\title{
Processing of higher count rates in Troitsk nu-mass experiment
}

\author{
Alexander Nozik ${ }^{1,2, \star}$ and Vaslily Chernov ${ }^{1}$ \\ ${ }^{1}$ Institute for Nuclear Research RAS, prospekt 60-letiya Oktyabrya 7a, Moscow 117312 \\ ${ }^{2}$ Moscow Institute of Physics and Technology, 9 Institutskiy per., Dolgoprudny, Moscow Region, 141700, \\ Russian Federation
}

\begin{abstract}
In this article we give a short outline of current status of search for sterile neutrinos with masses up to $4 \mathrm{keV}$ in "Troitsk nu-mass experiment". We also discuss major sources of systematic uncertainties and methods to lower them.
\end{abstract}

\section{Introduction}

Neutrino studies are one of the most motivating research areas in modern particle physics. While the particle itself was theoretically proposed in 1930-s, neutrino exact properties are still not known. Due to very small interaction cross-section, neutrino proved to be very hard to study experimentally. For now, one of the most interesting question is the absolute mass scale of neutrinos. The discovery of neutrino oscillations points to the fact that neutrino mass is not zero, but direct measurements still provide only upper limits for its mass of about $2 \mathrm{eV}$ ([1]).

Additional point of interest is the search for so-called sterile neutrinos (special type of neutrino that does not participate in weak interaction). There is a number of indirect indications that such sterile neutrinos could exist ([2]), but there is almost no direct knowledge about specific region of masses or mixing parameters.

\section{Troitsk nu-mass experiment}

Troitsk nu-mass experiment detailed description as well as sterile neutrino program proposal could be found in [3]. The recent results for sterile neutrino with mass up to $1 \mathrm{keV}$ are published in [4].

The main complication of search for sterile neutrino compared to search for electron neutrino mass performed previously in Troitsk nu-mass experiment ([1][5]) is the broader energy region. It produces two additional problems:

- The uncertainty caused by imprecise knowledge of electronics dead time.

- The uncertainty due to different number of events under the detector threshold for different retarding potentials.

The second problem is caused by back scattering effects discussed in [6] and could be solved only with precise simulations. In this article we will discuss the first problem and ways to solve it.

\footnotetext{
^e-mail:nozik@inr.ru
} 


\section{Reducing dead time uncertainty}

The current DAQ hardware, used in the Troitsk nu-mass has a dead time of approximately $6.5 \mu s$. It was sufficient for count rates of about $100 \mathrm{~Hz}$ used when the spectrum endpoint was investigated in search for electron neutrino mass ([1]). However, at count rates required to get sufficient statistics for sterile neutrino search $(20-40 \mathrm{kHz})$ even small deviations of estimated dead time could produce huge differences in count rate (see systematic uncertainties estimation in [7]). Furthermore, since dead time was determined by analog readout system, it could be precisely estimated only experimentally.

\subsection{Continuous digitization}

One of possible solutions to reduce the impact of dead time uncertainty is to reduce dead time itself. It could be done either by using faster electronics, or by using advanced signal shape analysis to designate pileup events in off-line analysis. The first approach is currently impossible to implement on Troitsk nu-mass because shorter shaping times introduce additional systematic error caused by additional events from detector back scattering (discussed in [6]). Therefore it was decided to switch to continuous digitization without changing shaping time.

There were two candidates for digitization hardware: CAEN DT-5270 and Rudnev-Shilaev Lan1012PCI. DT-5270 allowed for much faster sampling rate (31.25 MHz and higher), but it proved to be unsuitable for continuous digitization due to limitations of computer interface (USB channel bandwidth limit of $30 \mathrm{mb} / \mathrm{s}$ ). Lan10-12PCI, on the other hand, provides much lower sampling rate and have direct PCI-express interface with the computer bus. It was discovered that optimal Lan10-12PCI performance could be obtained with sampling rate of $3.125 \mathrm{MHz}$. In that case live time losses due to data download to computer amounts only to about $13 \%$.

Additional benefit of continuous digitization is the ability to simulate exact pile up effect using known signal shape. In order to do so one need to introduce an artificial signal and background noise distribution indistinguishable from real experimental data. In order to check the quality of analytic signal shape as well as background distribution, we plotted the distribution of $\chi^{2}$ over degree of freedom for experimental and simulated data versus analytic signal shape in Fig. 1.

It could be clearly seen, that both shapes are very similar and produce very good average quality of fit.

Three different algorithms were considered for off-line analysis of the signal shape:

1. Direct search for local peaks. This is the simplest and fastest method. However, it has rather high miss rate for close events because one needs a clear local minimum between events to separate them.

2. Search for local peaks with compensation. This method is a modification of the first one. In this case when the peak is found, it is reconstructed using analytic signal shape and subtracted from the initial signal to reduce influence on subsequent peaks.

3. Signal front fit. In this case reconstruction of signal performed not by finding its peak, but by fitting its front with analytical shape.

The results of simulations of different methods effectiveness for count rate about $40 \mathrm{kHz}$ are shown in table 1. Front fit method, as expected, gives better results for pileup recognition, but it produces a lot of false positive signals. Also it gives much worse performance then other two. Both methods 1 and 2 give similar effectiveness and performance. Further tests showed that using either of these methods produce an effective dead time caused by indistinguishable pile-up events of about $2.1 \mu \mathrm{s}$ 


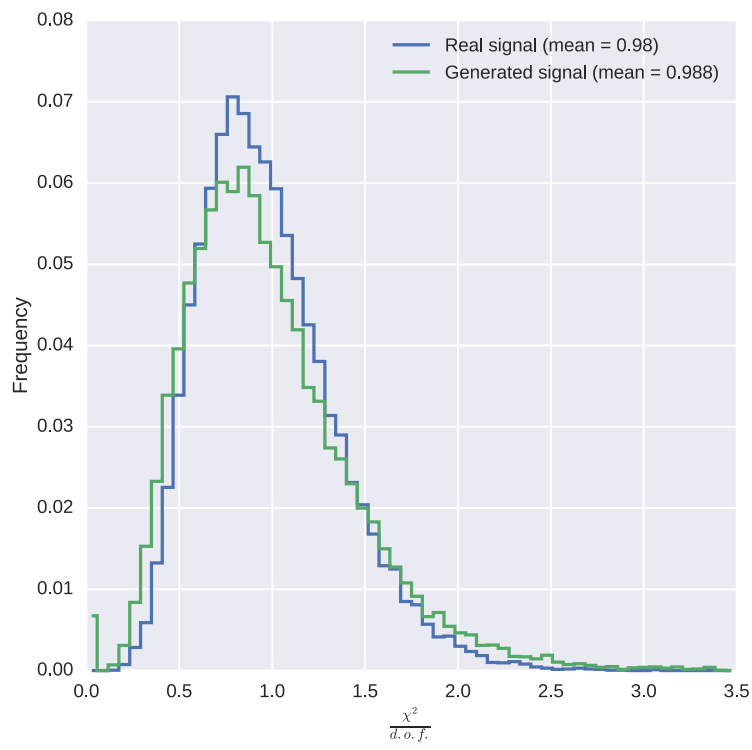

Figure 1: Distributions of $\chi^{2}$ over degree of freedom for simulated and experimental events versus analytic signal shape.

\begin{tabular}{|c|c|c|c|}
\hline & Direct & Direct with correction & Front fit \\
\hline Computation time, $\mathrm{s}$ & 1.222999 & 3.176033 & 195.431107 \\
\hline Total events & 80526 & 80526 & 80526 \\
\hline Recognized events & 72174 & 72244 & 79067 \\
\hline False negatives & 1358 & 1269 & 467 \\
\hline False positives & 6 & 4 & 1767 \\
\hline Pileup & 6467 & 6470 & 2607 \\
\hline
\end{tabular}

Table 1: Comparison of different peak recognition methods

which is almost 3 times better than the dead time of analog system. Furthermore, computation time of 1-3 s for $2 \mathrm{~s}$ measurement after optimization will allow to use these algorithms on-line to reduce stored data size.

\subsection{Statistical approach}

Another approach to dead time problem is to use information on events time distribution. The distribution of time delays between signals follows exponential distribution:

$$
f(t)=\frac{1}{\tau} e^{-t / \tau}
$$

where $\tau$ is the average time between events and $1 / \tau$ is the count rate. 


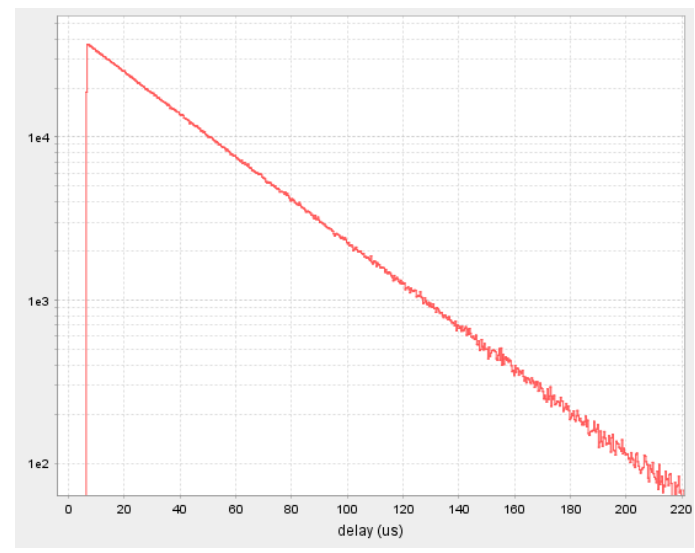

(a) Histogram of delays between events for dead time of $6.5 \mu s$.

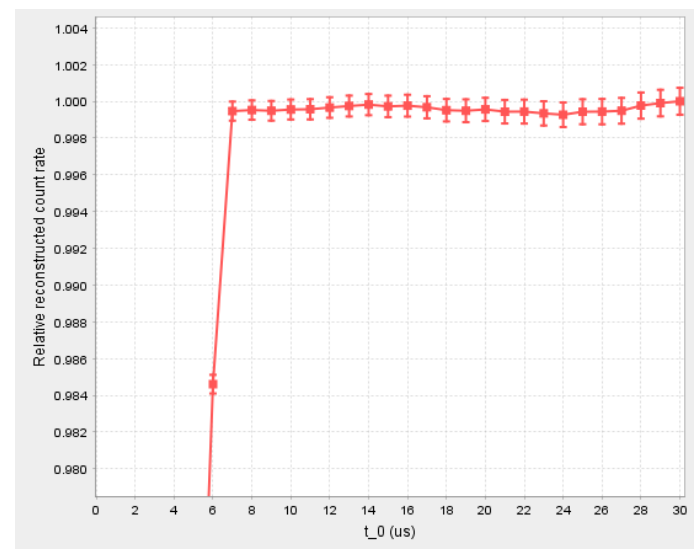

(b) Reconstructed count rate with error for different cutoff times $t_{0}$ (in $\mu s$.)

Figure 2: Statistical count rate reconstruction of simulated data.

Let us consider that there is sharp dead time margin $t_{0}$ that does not allow two events to be closer in time than this $t_{0}$. In this case the shape of time distribution above $t_{0}$ wont change, but there will be change in distribution normalization:

$$
f^{*}(t)=\frac{e^{t_{0} / \tau}}{\tau}\left\{\begin{array}{cc}
e^{-t / \tau} & t \geq t_{0} \\
0 & t<t_{0}
\end{array}\right.
$$

In order to avoid uncertainties from electronics dead time, one could select a $t_{0}$ slightly above the apparatus cutoff and estimate $\tau$ using modified distribution $f^{*}(t)$. Since $t_{0}$ is artificial, it could be selected with any needed precision and does not produce additional systematic error. Of course statistical error will be slightly increased because some events with delay below $t_{0}$ are excluded from analysis.

To get estimate of $\tau$ one can use the maximum likelihood method. The likelihood function for modified distribution could be written in a following way:

$$
L(\tau)=\prod_{i=1}^{N} f\left(t_{i}\right)=\left(\frac{e^{t_{0} / \tau}}{\tau}\right)^{N} \exp \left(-\frac{1}{\tau} \sum_{i=1}^{N} t_{i}\right),
$$

where $t_{i}$ are experimental times between events greater then $t_{0}$ and $N$ is total number of events with $t>t_{0}$. Designating $\sum_{i=1}^{N} t_{i}$ as $T$, the likelihood logarithm could be written in a following way:

$$
\ln L(\tau) \sim-N \ln \tau-\frac{1}{\tau}\left(T-N t_{0}\right)
$$

The maximum of $L(\tau)$ corresponds to

$$
\tau_{0}=\frac{T}{N}-t_{0}
$$

The difference between regular exponential distribution and modified one is additional shift $t_{0}$. The statistical uncertainty for $\tau_{0}$ is defined by the same formula as for regular one $\sigma_{\tau_{0}}=\tau_{0} / \sqrt{N}$. The 
ratio between statistical error for the whole data set and for cut one equals the square root of ratio between total number of events before and after the cut. For count rates up to $30-40 \mathrm{kHz}$ and $t_{0}$ about 5-10 $\mu s$ one can completely eradicate systematic error from the dead time, sacrificing only $10-15 \%$ of statistic sensitivity.

Fig. 2a shows the histogram of delays between subsequent events for a count rate of $30 \mathrm{kHz}$ with a dead time of $6.5 \mu \mathrm{s}$. Fig. $2 \mathrm{~b}$ shows count rate reconstructed via statistical procedure for different values of $t_{0}$. It could be clearly seen, that for $t_{0}$ above real dead time, the value of reconstructed rate suffers only slight fluctuations well within statistical errors and for higher values of $t_{0}$ errors are actually larger.

\section{Conclusion}

The combination of continuous digitization and statistical count rate estimation allow to significantly decrease the systematic error from dead time. The second primary source of uncertainty: events under the threshold, requires additional study and simulations. Algorithms used to distinguish pileup events could be optimized to be used on-line, thus allowing to use continuous digitization not only for one-pixel detector, but also for detectors with a lot of pixels without developing complicated storage system.

The work was supported by RFBR grant 17-02-00361 A. Authors would like to thank the whole Troitsk nu-mass group and particularly its leader Vladislav Pantuev.

\section{References}

[1] V.N. Aseev et al. (Troitsk), Phys. Rev. D84, 112003 (2011), 1108. 5034

[2] R. Adhikari et al., Submitted to: White paper (2016), 1602.04816

[3] D. Abdurashitov, A. Belesev, A. Berlev, V. Chernov, E. Geraskin, A. Golubev, G. Koroteev, N. Likhovid, A. Lokhov, A. Markin et al., arXiv preprint arXiv:1504.00544 (2015)

[4] J.N. Abdurashitov et al., JETP Lett. 105, 753 (2017), 1703 . 10779

[5] A.I. Belesev, A.I. Berlev, E.V. Geraskin, A.A. Golubev, N.A. Likhovid, A.A. Nozik, V.S. Pantuev, V.I. Parfenov, A.K. Skasyrskaya, J. Phys. G41, 015001 (2014), 1307. 5687

[6] P.V. Grigorieva, A.A. Nozik, V.S. Pantuev, A.K. Skasyrskaya (2015), 1511.06129

[7] D.N. Abdurashitov et al., JINST 10, T10005 (2015), 1504.00544 